\title{
SOME REMARKS OF DROP PROPERTY
}

\author{
PEI-KEE LIN
}

(Communicated by William J. Davis)

\begin{abstract}
Let $C$ be a proper closed convex set. $C$ is said to have the drop property if for any nonempty closed set $A$ disjoint with $C$, there is $a \in A$ such that $\operatorname{co}(a, C) \cap A=\{a\}$. We show that if $X$ contains a noncompact set with the drop property, then $X$ is reflexive. Moreover, we prove that if $C$ is a noncompact closed convex subset of a reflexive Banach space, then $C$ has the drop property if and only if $C$ satisfies the following conditions: (i) the interior of $C$ is nonempty; (ii) $C$ does not have any asymptote, and the boundary of $C$ does not contain any ray; and (iii) every support point $x$ of $C$ is a point of continuity.
\end{abstract}

\section{INTRODUCTION}

Let $(X,\|\cdot\|)$ be a real Banach space, and let $C$ be a nonempty proper closed convex subset of $X$. For any $x \notin C$, the drop determined by $x$ is the set $D(x, C)=\operatorname{co}(x, C)$, the convex hull of the set $\{x\} \cup C$. Daneš [D] proved that if $C$ is a bounded closed subset of $X$ and $A$ is a closed set at positive distance from $C$, then there exists an $a \in A$ such that $D(a, C) \cap A=\{a\}$. Modifying the assumption, Rolewicz [R1] said a nonempty proper closed set $C$ has the drop property if for every nonempty closed set $A$ disjoint with $C$, there exists a point $a \in A$ such that $D(a, C) \cap A=\{a\}$. The bounded closed convex sets with the drop property are studied in $[\mathrm{K} 1, \mathrm{~K} 2, \mathrm{M}, \mathrm{R} 1$, $\mathrm{R} 2]$. In [R1] Rolewicz proved that if the closed unit ball of $X$ has the drop property (in this case, we say $X$ has the drop property), then $X$ is reflexive. Kutzarova [K1] extended this result by showing $X$ is reflexive if $X$ contains a noncompact bounded closed convex set (respectively, a noncompact balanced closed convex set) with the drop property. Recently, Kutzarova and Rolewicz [KR1] showed that $X$ is reflexive if $X$ contains a noncompact closed convex symmetric set with the drop property.

For any subset $C$ of $X$, the Kuratowski measure of $C$ is the infimum $\alpha(C)$ of those $\varepsilon>0$ for which there is a covering of $C$ by a finite number of sets of diameter less then $\varepsilon$. It is known that $\alpha(C)=0$ if and only if $C$ is totally bounded. Let $C$ be a closed convex subset of $X$. We denote the set of all nonzero linear functionals $f \in X^{*}$, which are bounded above $C$ by $F(C)$.

Received by the editors December 7, 1990.

1980 Mathematics Subject Classification (1985 Revision). Primary 46B20, 46B10.

Key words and phrases. Drop property, reflexive space, compact, nearly uniformly convex. 
For any $f \in F(C)$, and any $\delta>0$, the slice $S(f, C, \delta)$ is the set

$$
\{x \in C: f(x) \geq M-\delta\} \text {, }
$$

where $M=\sup \{f(x): x \in C\}$. A closed convex set $C$ is said to have property $(\alpha)$ if

$$
\lim _{\delta \rightarrow 0} \alpha(S(f, C, \delta))=0
$$

for all $f \in F(C)$. It is easy to see that a closed convex set $C$ has property $(\alpha)$ if and only if for any $f \in F(C)$ and $x_{n} \in S\left(f, C, \frac{1}{n}\right),\left\{x_{n}\right\}$ contains a convergent subsequence. In [KR1] Kutzarova and Rolewicz proved the following

Theorem A. Let $C$ be any closed convex subset of $X$.

(i) If $C$ has the drop property, then $C$ has property $(\alpha)$.

(ii) If $C$ is not compact and if $C$ has the drop property, then $C$ has nonempty interior.

(iii) Suppose $X$ is reflexive. If $C$ has nonempty interior and $C$ has property $(\alpha)$, then $C$ has the drop property.

(iv) Let $C$ be a closed bounded convex set of a reflexive Banach space. If $\operatorname{int}(C) \neq \varnothing$ (where $\operatorname{int}(C)$ is the interior of $C$ ) and every support point of $C$ is a point of continuity, then $C$ has drop property.

Using Theorem $\mathrm{A}$, they proved that if $C_{1}$ and $C_{2}$ are any two bounded sets with the drop property, then $C_{1} \cap C_{2}, C_{1}+C_{2}$, and $\operatorname{co}\left(C_{1}, C_{2}\right)$ have the drop property. In $\S 2$ we show the assumption of boundedness can be removed. Hence, if $X$ contains a noncompact closed convex set with the drop property, then $X$ is reflexive. This gives an answer to a question of D. N. Kutzarova and S. Rolewicz [KR1].

Let $C$ be a closed convex set. $C$ is said to have property (*) if $C$ contains the ray $\{c+\lambda b: \lambda \geq 0\}$ implies for any $x \in X$, there is $\beta>0$ such that $x+(\beta+\lambda) b \in C$ for every $\lambda \geq 0$. In $\S 2$ we prove that if $C$ is a noncompact proper closed convex set of a reflexive Banach space, then $C$ has the drop property if and only if $\operatorname{int}(C) \neq \varnothing, C$ has property $(*)$, and every support point of $C$ is a point of continuity. This gives an extension of Theorem A(iv).

Recall a space $X$ is said to have the Kadec-Klee property (or property $(\mathrm{H})$ ) if on the unit sphere the weakly convergent sequence is convergent in norm (i.e., if $\left\|x_{n}\right\|=1$ and $x_{n}$ converges weakly to a unit vector $x$, then $x_{n}$ converges to $x$ in norm). V. Montesinos [M] proved that $X$ has the drop property if and only if $X$ is reflexive and $X$ has the Kadec-Klee property. Recall that a sequence $\left\{x_{n}\right\}$ is said to be an $\epsilon$-separate sequence for some $\epsilon>0$ if $\operatorname{sep}\left(x_{n}\right)=$ $\inf \left\{\left\|x_{n}-x_{m}\right\|: n \neq m\right\}>\epsilon$. A Banach space $X$ is said to have the uniform Kadec-Klee property if for every $\epsilon>0$ there is a $\delta>0$ such that if $x$ is a weak limit of a norm one $\epsilon$-separate sequence, then $\|x\|<1-\delta$. A Banach space is said to be nearly uniformly convex (NUC) if for every $\epsilon>0$ there exists a $\delta, 1>\delta>0$, such that for every sequence $\left\{x_{n}\right\} \subseteq B$ with $\operatorname{sep}\left(x_{n}\right)>\epsilon$, we have $\operatorname{co}\left(x_{n}\right) \cap(1-\delta) B \neq \varnothing$. It is easy to see that every (NUC) space has the uniform Kadec-Klee property, and every Banach space with the uniform Kadec-Klee property has the Kadec-Klee property. Huff $[\mathrm{H}]$ proved that $X$ is (NUC) if and only if $X$ is reflexive and $X$ has the uniform Kadec-Klee property. Modifying the theorem, Kutzarova and Rolewicz [KR2] said a closed convex set is (NUC) (respectively $\left(\mathrm{NUC}^{\prime}\right)$ ) with respect to a center $c \in C$ if 
for every $\epsilon>0$ there exists a $\delta, 1>\delta>0$, such that for every $\varepsilon$-separate sequence $\left\{x_{n}\right\} \subseteq C$

$$
\begin{gathered}
\operatorname{co}\left(x_{n}\right) \cap(1-\delta)(C-c) \neq \varnothing \\
\text { (respectively, } \left.\overline{\operatorname{co}}\left(x_{n}\right) \cap(1-\delta)(C-c) \neq \varnothing\right) .
\end{gathered}
$$

It is easy to see that if $C$ is (NUC) with respect to $c \in C$, then $C$ is $\left(\mathrm{NUC}^{\prime}\right)$ with respect to $c$. Kutzarova and Rolewicz [KR2] proved that if $c$ is an interior point of $C$, then $C$ is (NUC) with respect to $c$ if and only if $C$ is $\left(\mathrm{NUC}^{\prime}\right)$ with respect to any $c$. They asked whether this is still true if $c$ is a boundary point of $C$. In $\S 3$, we show this is true if $C$ has the drop property. We also give an example to show the assumption of the drop property cannot be removed.

\section{ON THE DROP PROPERTY}

In [KR1] Kutzarova and Rolewicz asked whether $X$ is reflexive if $X$ contains a noncompact closed convex with the drop property. The following theorem shows the answer is affirmative.

Theorem 1. Let $C_{1}$ and $C_{2}$ be any two closed convex subsets of $X$ with the drop property. If $C_{1} \cap C_{2} \neq \varnothing$, then $C_{1} \cap C_{2}$ has the drop property. Hence, if $X$ contains a noncompact closed convex set with the drop property, then $X$ is reflexive.

Proof. Let $A$ be any closed subset of $X$ such that $A \cap\left(C_{1} \cap C_{2}\right)=\varnothing$. If $A \cap C_{1}=\varnothing$, then there exists $a \in A$ such that $D\left(a, C_{1}\right) \cap A=\{a\}$. This implies $D\left(a,\left(C_{1} \cap C_{2}\right)\right) \cap A=\{a\}$. So we may assume that $A \cap C_{1} \neq \varnothing$. Since $\left(A \cap C_{1}\right) \cap C_{2}=\varnothing$ and $C_{2}$ has the drop property, there is $a \in A \cap C_{1}$ such that $D\left(a, C_{2}\right) \cap\left(A \cap C_{1}\right)=\{a\}$. So

$$
D\left(a,\left(C_{2} \cap C_{1}\right)\right) \cap A \subseteq\left(D\left(a,\left(C_{2} \cap C_{1}\right)\right) \cap C_{1}\right) \cap A=\{a\},
$$

and $C_{1} \cap C_{2}$ has the drop property.

It is easy to see that if $X$ contains a noncompact closed convex set with the drop property, then $X$ contains a noncompact symmetric closed convex set with the drop property. By [KR1, Proposition 4], $X$ is a reflexive space.

Remark 1. Let $C$ be an unbounded closed convex set of a reflexive space. Kutzarova and Rolewicz proved that if $S(f, C, 1)$ is bounded for some $f \in$ $F(C)$, then $C$ contains a ray $\{c+\beta b: \beta>0\}$. Moreover, if $c^{\prime} \in C, C$ also contains the ray $\left\{c^{\prime}+\beta b: \beta>0\right\}$.

Let $C_{1}$ and $C_{2}$ be any two bounded closed convex sets with the drop property. In [KR1] Kutzarova and Rolewicz proved that $\lambda C_{1}+\mu C_{2}$ and $\operatorname{co}\left(C_{1}, C_{2}\right)$ have the drop property. The following theorem shows that the boundedness can be removed.

Theorem 2. Let $C_{1}$ and $C_{2}$ be any two closed convex sets with the drop property. If $\operatorname{co}\left(C_{1}, C_{2}\right) \neq X$, then

(i) for any $\lambda, \mu \neq 0, \lambda C_{1}+\mu C_{2}$ is closed, and it has the drop property;

(ii) $\operatorname{co}\left(C_{1}, C_{2}\right)$ is closed, and it has the drop property.

Proof. We only prove (ii) and leave the proof of (i) to the reader. If $C_{1}$ and $C_{2}$ are compact, then $\operatorname{co}\left(C_{1}, C_{2}\right)$ is compact. So we may assume that $\operatorname{co}\left(C_{1}, C_{2}\right)$ has an interior point. 
First, we show $\operatorname{co}\left(C_{1}, C_{2}\right)$ has property $(\alpha)$. If $f \in F\left(\operatorname{co}\left(C_{1}, C_{2}\right)\right)$, then $f \in F\left(C_{1}\right) \cap F\left(C_{2}\right)$. Let $x$ (respectively, $x^{\prime}$ ) be a point in $C_{1}$ (respectively, $C_{2}$ ) such that

$$
\begin{gathered}
f(x)=\sup \left\{f(y): y \in C_{1}\right\} \\
\text { (respectively, } \left.f\left(x^{\prime}\right)=\sup \left\{f(y): y \in C_{2}\right)\right\} .
\end{gathered}
$$

One can easily show that

$$
S\left(f, \operatorname{co}\left(C_{1}, C_{2}\right), \delta\right)=\operatorname{co}\left(x, x^{\prime}\right)+\left(S\left(f, C_{1}, \delta\right)-x\right)+\left(S\left(f, C_{2}, \delta\right)-x^{\prime}\right) .
$$

(Compare with the proof of [KR1, Theorem 9 (iii)].) So

$$
\lim _{\delta \rightarrow 0} \alpha\left(S\left(f, \operatorname{co}\left(C_{1}, C_{2}\right), \delta\right)=0\right.
$$

and

$$
\operatorname{co}\left(C_{1}, C_{2}\right) \text { has property }(\alpha) \text {. }
$$

Suppose that $b \in \overline{\operatorname{co}\left(C_{1}, C_{2}\right)} \backslash \operatorname{co}\left(C_{1}, C_{2}\right) \neq \varnothing$. By Hahn-Banach Theorem, there is a linear functional $f$ such that $f(b) \geq f(x)$ for all $x \in \operatorname{co}\left(C_{1}, C_{2}\right)$. Since $b \in \overline{\operatorname{co}\left(C_{1}, C_{2}\right)}$ there exist $x_{n} \in C_{1}, x_{n}^{\prime} \in C_{2}$, and $0 \leq \beta_{n} \leq 1$ such that

$$
\lim _{n \rightarrow \infty} \beta_{n} x_{n}+\left(1-\beta_{n}\right) x_{n}^{\prime}=b .
$$

By passing to a subsequece, we may assume that $\left\{\beta_{n}\right\}$ converges to some $\beta$, $0 \leq \beta \leq 1$. It is easy to see that if $\beta \neq 0$ (respectively, $\beta \neq 1$ ), then

$$
\begin{gathered}
\lim _{n \rightarrow \infty} f\left(x_{n}\right)=\sup \left\{f(y): y \in C_{1}\right\} \\
\text { (respectively, } \lim _{n \rightarrow \infty} f\left(x_{n}^{\prime}\right)=\sup \left\{f(y): y \in C_{2}\right\} \text { ). }
\end{gathered}
$$

But $C_{1}$ and $C_{2}$ have the drop property. Hence, if $\beta \neq 0$ (respectively, $\beta \neq 1$ ), then $\left\{x_{n}\right\}$ (respectively, $\left\{x_{n}^{\prime}\right\}$ ) contains a subsequence that converges to some element

$$
x \in\left\{y \in C_{1}: f(y)=\sup \left\{f(z): z \in C_{1}\right\}(=f(b))\right\}
$$

(respectively, $x^{\prime} \in\left\{y \in C_{2}: f(y)=\sup \left\{f(z): z \in C_{2}\right\}(=f(b))\right\}$ ).

So if $0<\beta<1$, then $b=\beta x+(1-\beta) x^{\prime} \in \operatorname{co}\left(C_{1}, C_{2}\right)$. On the other hand, if $\beta=1$ (respectively, $\beta=0$ ), then

$$
\begin{gathered}
b=\lim _{n \rightarrow \infty}\left(\beta_{n} x+\left(1-\beta_{n}\right) x_{n}^{\prime}\right) \in \overline{D\left(x, C_{2}\right)} \\
\text { (respectively, } \left.b=\lim _{n \rightarrow \infty}\left(\beta_{n} x_{n}+\left(1-\beta_{n}\right) x^{\prime}\right) \in \overline{D\left(x^{\prime}, C_{1}\right)}\right) .
\end{gathered}
$$

By Proposition 5 of [KR1], $D\left(x, C_{2}\right)$ and $D\left(x^{\prime}, C_{1}\right)$ are closed sets. So $b \in$ $\operatorname{co}\left(C_{1}, C_{2}\right)$; we get a contradiction.

Lemma 3. Let $C$ be a closed convex set with nonempty interior. If $C$ has property $(\alpha)$, then $C$ has property $(*)$.

Proof. Suppose it is not true. There exist $c \in C$ and $b, x \in X$ such that $b \neq 0$, $\{c+\lambda b: \lambda \geq 0\} \subseteq C$ but $\{x+\lambda b: \lambda \geq 0\} \cap C$ is not a ray. By the simple convexity argument (see [KR1, Proof of Lemma 2]), the line $\{x+\lambda b: \lambda \in \mathbb{R}\}$ 
is disjoint with $C$. Since $C$ has at least one interior point, by Hahn-Banach Theorem, there is $f \in X^{*}$ such that

$$
\inf \{f(x+\lambda b): \lambda \in \mathbb{R}\} \geq M=\sup \{f(y): y \in C\} .
$$

This implies $f(b)=0$, and $S(f, C, M-f(c)+1)$ contains a ray. We get a contradiction and $C$ must have property $(*)$.

Remark 2. Let $C$ be a closed convex subset of $X$. A ray $r=\{x+\lambda y: \lambda>0\}$ is said to be an asymptote if $r \cap C=\varnothing$, and for any $\epsilon>0$ there is $N>0$ such that $\lambda>N$ implies $d(x+\lambda y, C)=\inf \{\|x+\lambda y-c\|: c \in C\}<\epsilon$. Suppose $C$ is a closed convex set with nonempty interior. Then $C$ has property (*) if and only if $C$ does not have any asymptote and the boundary of $C$ does not contain any ray. The proof is left to the reader.

Let $C$ be a closed convex set. $c \in C$ is said to be a support point of $C$ if there exists $f \in X^{*}, f \neq 0$, such that $f(c)=\sup \{f(x): x \in C\}$. A point $c$ in $C$ is said to be a point of continuity if for every sequence $\left\{x_{n}\right\}$ in $C,\left\{x_{n}\right\}$ converges to $c$ weakly implies $\left\{x_{n}\right\}$ converges to $c$ in norm.

Theorem 4. Let $C$ be a noncompact closed convex subset of a reflexive Banach space. Then the following are equivalent.

(i) $C$ has the drop property;

(ii) $\operatorname{int}(C) \neq \varnothing$ and $C$ has property $(\alpha)$;

(iii) $\operatorname{int}(C) \neq \varnothing, C$ has property $(*)$, and every support point $x$ of $C$ is a point of continuity.

Proof. By Theorem A and Lemma 3, we only need to show (iii) implies (ii). First, we claim that for each $f \in F(C), S(f, C, \delta)$ is bounded. Suppose it is not true. There exist $f \in F(C)$ and $\left\{x_{n}\right\} \subseteq C$ such that $\lim _{n \rightarrow \infty}\left\|x_{n}\right\|=\infty$ and $\lim _{n \rightarrow \infty} f\left(x_{n}\right)=M=\sup \{f(x): x \in C\}$. Let $y$ be any vector in $X$ such that $\|y\|<2$ and $f(y)=1$.

Case 1. There is a subsequence of $\left\{x_{n} /\left\|x_{n}\right\|\right\}$ that conveges weakly to a nonzero vector $b \in X$. Then $r=\left\{x_{1}+\lambda b: \lambda \geq 0\right\} \subseteq C$ and $f(b)=$ $\lim _{n \rightarrow \infty} f\left(x_{n}\right) /\left\|x_{n}\right\|=0$, but the ray $\{(M+1) y+\lambda b: \lambda>0\}$ is disjoint with $C$. We get a contradiction.

Case 2. The $\left\{x_{n} /\left\|x_{n}\right\|\right\}$ converges weakly to 0 . Without loss of generality, we may assume that 0 is on the boundary of $C$. So $\left\{x_{n} /\left\|x_{n}\right\|\right\}$ converges to 0 in norm. This is impossible, and we prove our claim.

Let $x_{n}$ be any point in $S\left(f, C, \frac{1}{n}\right)$. Since $X$ is reflexive, $\left\{x_{n}\right\}$ contains a weakly convergent subsequence $\left\{x_{n_{k}}\right\}$, say it converges to $y \in C$ weakly. Clearly, $f(y)=\sup \{f(x) ; x \in C\}$. So $y$ is a support point, and $\left\{x_{n_{k}}\right\}$ converges to $y$. This implies $C$ has property $(\alpha)$.

\section{NEARLY UNIFORM CONVEXITY}

Recall a closed convex set is said to be (NUC ${ }^{\prime}$ ) with a center $a$ if for every $\epsilon>0$ there exists a $\delta, 1>\delta>0$ such that for every $\epsilon$-separate sequence in $C, \overline{\mathrm{co}}\left(x_{n}\right) \cap(a+(1-\delta)(C-a)) \neq \varnothing$. It is easy to see that if $C$ is (NUC) with respect to an $a \in \operatorname{int}(C)$ if and only if $C$ is (NUC') with respect to $a$. In [KR2] D. N. Kutzarova and S. Rolewicz asked whether (NUC) and (NUC ') are equivalent. The following theorem shows the answer is affirmative if $C$ has the drop property. 
Theorem 5. Let $C$ be a closed convex set with the drop property and $c \in C$. Then $C$ is (NUC) with respect to $c$ if (and only if) $C$ is $\left(N U C^{\prime}\right)$ with respect to $c$.

Proof. Since every compact convex set is (NUC), we may assume that the interior of $C$ is nonempty. Let $\left\{x_{n}\right\}$ be an $\epsilon$-seperate sequence in $C$. If $\left\{x_{n}\right\}$ is not bounded, then $\overline{\mathrm{co}}\left(x_{n}\right)$ contains the ray $r=\left\{x_{1}+\lambda b: \lambda \geq 0\right\}$ for some $b \neq 0$. By Lemma 3 , there exists $\beta>0$ such that $c+2\left(x_{1}-c+\frac{\beta}{2} b\right)=$ $c+2\left(x_{1}-c\right)+\beta b \in \operatorname{int}(C)$. So $x_{1}+\frac{\beta}{2} b \in \overline{\operatorname{co}}\left(x_{n}\right) \cap \operatorname{int}\left(c+\frac{1}{2}(C-c)\right) \neq \varnothing$.

If $\left\{x_{n}\right\}$ is bounded, then by passing to a subsequence we may assume $\left(x_{n}\right)$ converges weakly, say it converges to $y \in c+(1-\delta)(C-c)$ weakly. Since $C$ has the drop property, $y$ is an interior point of $C$. This implies $y \in$ $\operatorname{int}\left(c+\left(1-\frac{\delta}{2}\right)(C-c)\right)$ and we prove the theorem.

Remark 3. The proof of the above theorem shows that if $C$ has the drop property, then $C$ is (NUC) with respect to $c$ if and only if it satisfies the following condition:

(o) for any $\epsilon>0$, there is $\delta, 0<\delta<1$, such that if $x$ is a weak limit of an $\epsilon$-seperate sequence in $C$, then $x \in c+(1-\delta)(C-c)$.

The following example shows the drop property cannot be removed from the above theorem.

Example 1. Let $\left\{e_{n}\right\}$ be the natural basis of $\ell_{2}$, and let $C$ be the closed convex hull of $\left\{e_{n}: n \in \mathbb{N}\right\}$. Clearly, $0 \in C$. For any $0<\delta<1$ and for any $c \in \operatorname{co}\left\{e_{n}: n \in \mathbb{N}\right\},(1-\delta)^{-1} c \notin C$. So $C$ is not (NUC) with respect to 0 . We claim that if $x$ is a weak limit of an $\epsilon \sqrt{2}$-sepaeate sequence $\left\{x_{n}\right\} \subseteq C$, then $x \in(1-\epsilon) C$.

By passing to a subsequence and perturbing $\left(x_{n}\right)$, we may assume that there exists a block squence $\left\{z_{n}\right\}$ such that $x_{n}=x+z_{n}$ and $\left\|z_{n}\right\|_{2} \geq \epsilon$. But $\left\|z_{n}\right\|_{1} \geq\left\|z_{n}\right\|_{2}$. We have $x \in(1-\epsilon) C$. So $C$ is $\left(\right.$ NUC $\left.^{\prime}\right)$ with respect to 0 .

\section{REFERENCES}

[D] J. Daneš, A geometric theorem useful in nonlinear functional analysis, Boll. Un. Mat. Ital. (6) (1972), 369-375.

[Di] J. Diestel, Geometry of Banach spaces-selected topics, Lecture Notes in Math., vol. 485, Springer-Verlag, Berlin and Heidelberg, 1975.

[H] R. Huff, Banach spaces which are nearly uniformly convex, Rocky Mountain J. Math. 10 (1980), 743-749.

[K1] D. N. Kutzarova, A sufficient condition for the drop property, C. R. Acad. Bulgar Sci. 39 (1986), 17-19.

[K2] - On the drop property of convex sets in Banach spaces, Constructive Theory of Functions 1987,1988 , pp. 283-287.

[KR1] D. N. Kutzarova and S. Rolewicz, Drop property for convex sets, Arch. Math. 56 (1991), 501-511.

[KR2] - On nearly uniformly convex sets, Arch. Math. (Basel) (to appear).

[M] V. Montesinos, Drop property equals reflexivity, Studia Math. 87 (1987), 93-100.

[R1] S. Rolewicz, On drop property, Studia Math. 85 (1987), 27-35.

[R2] - On $\Delta$-uniform convexity and drop property, Studia Math. 87 (1987), 181-191.

Department of Mathematics, Memphis State Univeristy, Memphis, Tennessee 38152 